\title{
Measure-Theoretic Properties of Level Sets of Distance Functions
}

\author{
Daniel Kraft \\ University of Graz \\ Institute of Mathematics, NAWI Graz \\ Universitätsplatz 3, 8010 Graz, Austria \\ Email: daniel.kraft@uni-graz.at
}

May 21st, 2015

\begin{abstract}
We consider the level sets of distance functions from the point of view of geometric measure theory. This lays the foundation for further research that can be applied, among other uses, to the derivation of a shape calculus based on the level-set method. Particular focus is put on the $(n-1)$-dimensional Hausdorff measure of these level sets. We show that, starting from a bounded set, all sub-level sets of its distance function have finite perimeter. Furthermore, if a uniform density condition is satisfied for the initial set, one can even show an upper bound for the perimeter that is uniform for all level sets. Finally, we present an example that shows that our results are sharp in the sense that no uniform upper bound can exist if our density condition is not satisfied. This is even true if the initial set is otherwise very regular (i. e., a bounded Caccioppoli set with smooth boundary).
\end{abstract}

Keywords: Geometric Measure Theory, Level Set, Distance Function, Hausdorff Measure, Perimeter, Caccioppoli Set

\section{Introduction}

In this paper, we are interested in a measure-theoretic analysis of the level sets of distance functions. For some open set $\Omega_{0} \subset \mathbb{R}^{n}$, the distance function is given by

$$
d_{\Omega_{0}}(x)=\inf _{y \in \Omega_{0}}|x-y|
$$

This is a widely studied construct with well-known properties. See, for instance, chapter 6 of 6 . In particular, note that $d_{\Omega_{0}}(x)$ is well-defined and non-negative for all $x \in \Omega_{0}$. Furthermore, the function $d_{\Omega_{0}}$ is continuous on $\mathbb{R}^{n}$. For $t>0$, let us also define

$$
\begin{aligned}
& \Omega_{t}=d_{\Omega_{0}}{ }^{-1}((-\infty, t))=\left\{x \in \mathbb{R}^{n} \mid d_{\Omega_{0}}(x)<t\right\} \text { and } \\
& \Gamma_{t}=d_{\Omega_{0}}{ }^{-1}(\{t\})=\left\{x \in \mathbb{R}^{n} \mid d_{\Omega_{0}}(x)=t\right\} .
\end{aligned}
$$

Continuity of the distance function implies that $\Omega_{t}$ is open and $\Gamma_{t}$ closed. It is also easy to see that $\Gamma_{t}$ coincides with the topological boundary of $\Omega_{t}$. I. e., $\Gamma_{t}=\partial \Omega_{t}$. The set $\Omega_{t}$ is sometimes called $t$-envelope of $\Omega_{0}$ in the literature. It is an inflated and smoothed version of $\Omega_{0}$.

To see why the study of these sets is interesting, let us briefly mention the classical level-set method introduced by Osher and Sethian in 13]: Based on this method, one can describe evolving shapes as the sub-zero level sets of a time-dependent level-set function. If the geometry is changed by moving the boundary in normal direction according to a given speed field, the time evolution of the level-set function can be described by the so-called level-set equation. Based on this method, one can, for instance, build a framework for shape optimisation as done in [4]. See [8], 5] and [12 for some recent applications. Our work in 11 allows to represent the propagating domains in the level-set framework with a formula similar to (1) In the general case, one uses the solution of an Eikonal equation instead of the distance function $d_{\Omega_{0}}$. The situation considered here is a special case, which results if the speed field is positive and 
constant throughout all of $\mathbb{R}^{n}$. Furthermore, shape sensitivity analysis in this context leads naturally to the question whether or not the surface measure of $\Gamma_{t}$ (or, equivalently, the perimeter of $\Omega_{t}$ ) is continuous with respect to the propagation time $t$. One half of this question can be resolved quite easily by the wellknown lower semi-continuity property of the total variation. This, in turn, implies lower semi-continuity for the perimeter of the evolving sets. Estimates in the other direction, however, are more difficult to obtain.

It is the aim of this paper to explore one approach to establish such estimates. After investigating some auxiliary geometric properties of spherical sectors in Section 2, we will derive our main results in Section 3. The first is a kind of inverse isoperimetric inequality (see Theorem 2), that gives an upper bound on the perimeter $P\left(\Omega_{t}\right)$ of $\Omega_{t}$ in terms of the created volume $\Omega_{t} \backslash \Omega_{0}$. An obvious estimate of this volume follows if $\Omega_{0}$ is bounded, which results in Corollary 1. Note, however, that this only yields an upper bound for $P\left(\Omega_{t}\right)$ that diverges like $1 / t$ for $t \rightarrow 0^{+}$. Under an additional uniform density assumption on $\Omega_{0}$, we can further improve the estimate: In this situation, the volume can be bounded in terms of the perimeter of the initial domain $\Omega_{0}$ times $t$. Consequently, we obtain a uniform bound on the perimeter of $\Omega_{t}$. This will be done in Subsection 3.2. Subsection 3.3 discusses the required uniform density condition in comparison to related geometric properties in the literature. In the final Section 4. we give an example that demonstrates that our uniform density property is really necessary to get a uniform bound on $P\left(\Omega_{t}\right)$. If it is not fulfilled, the perimeter of $\Omega_{t}$ can diverge for $t \rightarrow 0^{+}$even if $\Omega_{0}$ is a smooth Caccioppoli set.

\section{Auxiliary Geometric Results}

In order to show our main results in Section 3 (in particular, Theorem 2), we need some auxiliary results. While they are only based on elementary geometry, their proofs are, nevertheless, quite technical. In this section, we will develop these results. The basic object studied is what we will call a sector below:

Definition 1. Let $x_{0}, x \in \mathbb{R}^{n}$ and $\phi \in[0, \pi / 2]$. We define

$$
S_{\phi}\left(x_{0}, x\right)=\left\{y \in \mathbb{R}^{n}|0<| x_{0}-y|<| x_{0}-x \mid \text { and }\left(y-x_{0}\right) \cdot\left(x-x_{0}\right)>\left|x_{0}-y\right|\left|x_{0}-x\right| \cdot \cos \phi\right\} .
$$

We will often set $t=\left|x_{0}-x\right|$ to be the sector's radius. For $0 \leq \underline{f}<\bar{f} \leq 1$, we further define

$$
S_{\phi}\left(x_{0}, x ; \underline{f}, \bar{f}\right)=\left\{y \in S_{\phi}\left(x_{0}, x\right)|\underline{f} t<| x_{0}-y \mid<\bar{f} t\right\} .
$$

$S_{\phi}\left(x_{0}, x\right)$ is an open sector of the ball with centre $x_{0}$ and radius $t=\left|x_{0}-x\right|$. The value of $\phi$, which corresponds to the maximum allowed angle $x-x_{0}-y$, defines the sector's aperture. The restricted version $S_{\phi}\left(x_{0}, x ; f, \bar{f}\right)$ is similar to a conic frustum, except that its bases are spherical instead of planar. One can easily see that

$$
S_{\phi}\left(x_{0}, x\right)=S_{\phi}\left(x_{0}, x ; 0,1\right)
$$

for the degenerate case $f=0$ and $\bar{f}=1$. Besides using the angle $\phi$ directly, we will also need to define such a sector via an auxiliary ball $B_{\delta}(x)$ for $\delta<t$. The idea is depicted in Figure 1b. In this case, the sector's aperture is defined indirectly via $\delta$. It is chosen as the angle at which the ball around $x$ intersects the larger sphere with centre $x_{0}$. With basic trigonometry, one can derive

$$
\phi(\delta)=\arccos \left(1-\frac{\delta^{2}}{2 t^{2}}\right)
$$

for the corresponding aperture angle. In the following, we will only need two basic properties of this explicit function: $\delta<t \phi(\delta)$ holds for all $\delta$ and $t \phi(\delta) / \delta \rightarrow 1$ in the limit $\delta \rightarrow 0^{+}$. In other words, $t \phi(\delta) \approx \delta$ asymptotically for small $\delta$.

\subsection{The Volume of Sectors}

The first part of our geometric analysis of sectors is concerned with determining their volume (i. e., $n$-dimensional Lebesgue measure). For this, let us state the following fundamental geometric facts: 


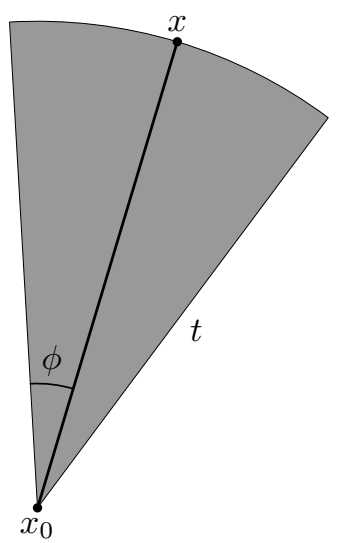

(a) Based on $\phi$ according to Definition 1

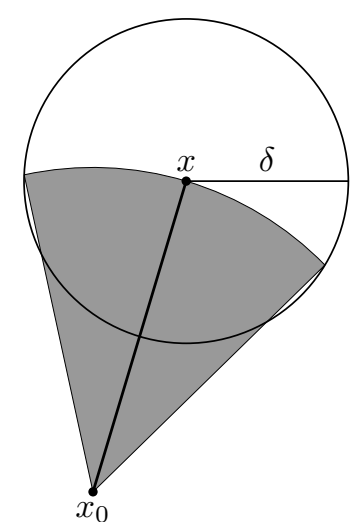

(b) Defined via the ball $B_{\delta}(x)$ and $(2)$

Figure 1: Definitions of the sector $S_{\phi}\left(x_{0}, x\right)$.

Lemma 1. Let $n \geq 2$. The volume of a ball with radius $\rho>0$ is given by

$$
\operatorname{vol}\left(B_{\rho}(x)\right)=\omega_{n} \rho^{n}, \quad \omega_{n}=\frac{\pi^{n / 2}}{\Gamma(n / 2+1)} .
$$

This holds obviously for arbitrary $x \in \mathbb{R}^{n}$.

Furthermore, there exists a mapping $r:[0, \pi / 2] \rightarrow[0,1 / 2]$ which is continuous, bijective, strictly increasing and satisfies

$$
\operatorname{vol}\left(S_{\phi}\left(x_{0}, x\right)\right)=r(\phi) \cdot \operatorname{vol}\left(B_{t}(0)\right)=r(\phi) \cdot \omega_{n} t^{n}
$$

for all $x_{0}, x \in \mathbb{R}^{n}$ and $\phi \in[0, \pi / 2]$. Here, we have set $t=\left|x_{0}-x\right|$ as before. In addition,

$$
\lim _{\phi \rightarrow 0^{+}} \frac{r(\phi)}{\phi^{n-1}}>0
$$

exists and is strictly positive.

Proof. The volume of $n$-dimensional balls is a well-known result. See, for instance, Theorem 26.13 in [14]. In order to compute the volume of $S_{\phi}\left(x_{0}, x\right)$, we use spherical coordinates:

$$
\begin{aligned}
y_{1} & =r \cos \phi_{1} \\
y_{k} & =r \prod_{i=1}^{k-1} \sin \phi_{i} \cdot \cos \phi_{k} \text { for } k=2, \ldots, n-2, \\
y_{n-1} & =r \prod_{i=1}^{n-2} \sin \phi_{i} \cdot \sin \phi_{n-1} \\
y_{n} & =r \prod_{i=1}^{n-2} \sin \phi_{i} \cdot \cos \phi_{n-1}
\end{aligned}
$$

For a derivation of spherical coordinates particularly suited to our purposes, see [2]. Based on the derivation given there, it is easy to see that

$$
S_{\phi}\left(x_{0}, x\right)=\left\{x_{0}+y \in \mathbb{R}^{n} \mid y=y\left(r, \phi_{1}, \ldots, \phi_{n-1}\right), r \in(0, t), \phi_{1} \in[0, \phi)\right\} .
$$

The other angles are drawn from their full range, i. e., $\phi_{k} \in[0, \pi]$ for $k=2, \ldots, n-2$ and $\phi_{n-1} \in[0,2 \pi)$. Since the Jacobian determinant of this transformation is

$$
\operatorname{det} J\left(r, \phi_{1}, \ldots, \phi_{n-1}\right)=r^{n-1} \prod_{i=1}^{n-2} \sin ^{n-i-1} \phi_{i},
$$


we find by simple integration that

$$
\operatorname{vol}\left(S_{\phi}\left(x_{0}, x\right)\right)=\int_{0}^{t} r^{n-1} d r \cdot \int_{0}^{\phi} \sin ^{n-2} \phi_{1} d \phi_{1} \cdot \prod_{i=2}^{n-2} \int_{0}^{\pi} \sin ^{n-i-1} \phi_{i} d \phi_{i} \cdot \int_{0}^{2 \pi} d \phi_{n-1} .
$$

Note that the integral over $r$ is trivial to compute. The integrals over $\phi_{2}, \ldots \phi_{n-1}$ result in a dimensional constant. If $\phi$ is chosen as $\pi$, we recover the full volume of $B_{t}\left(x_{0}\right)$. Thus,

$$
r(\phi)=\frac{\int_{0}^{\phi} \sin ^{n-2} \phi^{\prime} d \phi^{\prime}}{\int_{0}^{\pi} \sin ^{n-2} \phi^{\prime} d \phi^{\prime}}
$$

fulfils (3) All other claimed properties of $r$ follow from this expression.

Based on Lemma 1, we can also derive how the volume changes if we use a different angle $\phi$ and consider a frustum with non-degenerate $\underline{f}$ and $\bar{f}$ :

Lemma 2. Let $0 \leq \underline{f}<\bar{f} \leq 1$. Then

$$
\operatorname{vol}\left(S_{\phi}\left(x_{0}, x ; \underline{f}, \bar{f}\right)\right)=\operatorname{vol}\left(S_{\phi}\left(x_{0}, x\right)\right) \cdot\left(\bar{f}^{n}-\underline{f}^{n}\right)
$$

for arbitrary $x_{0}, x \in \mathbb{R}^{n}$ and $\phi \in[0, \pi / 2]$.

If $\phi^{\prime} \in[0, \pi / 2]$ in addition, then

$$
\operatorname{vol}\left(S_{\phi^{\prime}}\left(x_{0}, x\right)\right)=\frac{r\left(\phi^{\prime}\right)}{r(\phi)} \cdot \operatorname{vol}\left(S_{\phi}\left(x_{0}, x\right)\right) .
$$

In particular, for $\phi^{\prime}=a \phi$ with some fixed ratio $a>0$,

$$
\lim _{\phi \rightarrow 0^{+}} \frac{\operatorname{vol}\left(S_{a \phi}\left(x_{0}, x\right)\right)}{\operatorname{vol}\left(S_{\phi}\left(x_{0}, x\right)\right)}=a^{n-1} .
$$

This remains true if we consider $S_{\phi^{\prime}}\left(x_{0}, x ; \underline{f}, \bar{f}\right)$ instead of $S_{\phi^{\prime}}\left(x_{0}, x\right)$.

Proof. The first result follows immediately by subtracting the volumes of sectors with radii $\bar{f} t$ and $f t$. The second is a direct consequence of (3)] Finally, Lemma 1 also implies that

$$
\lim _{\phi \rightarrow 0^{+}} \frac{r(a \phi)}{r(\phi)}=a^{n-1} \cdot \lim _{\phi \rightarrow 0^{+}} \frac{r(a \phi)}{(a \phi)^{n-1}} \frac{\phi^{n-1}}{r(\phi)}=a^{n-1} .
$$

This completes the proof.

Note that the rate of convergence in the limit (5) does not depend on $x_{0}, x$ or $t$. In particular, this implies that for fixed $a$ and $n$, there exist constants $0<C^{\prime}<C^{\prime \prime}$ such that

$$
C^{\prime} \cdot \operatorname{vol}\left(S_{\phi}\left(x_{0}, x\right)\right) \leq \operatorname{vol}\left(S_{a \phi}\left(x_{0}, x\right)\right) \leq C^{\prime \prime} \cdot \operatorname{vol}\left(S_{\phi}\left(x_{0}, x\right)\right)
$$

for all $\phi$ small enough. The same is true for the frustum $S_{a \phi}\left(x_{0}, x ; f, \bar{f}\right)$.

Another conclusion from Lemma 1 is the following estimate, which relates, somehow, the surface area of a sector's base to its volume. This result will be used later when we prove Theorem 2

Lemma 3. For fixed $t>0$, there exist $\delta_{0}>0$ and a dimensional constant $C$ such that

$$
\delta^{n-1} \omega_{n-1} \leq C \frac{\operatorname{vol}\left(S_{\phi(\delta)}\left(x_{0}, x\right)\right)}{t}
$$

for all $\delta \in\left(0, \delta_{0}\right)$ and arbitrary $x_{0}, x \in \mathbb{R}^{n}$ with $\left|x_{0}-x\right|=t$.

Proof. By (4), there exist $\phi_{0}>0$ and $C^{\prime}$ such that $\phi^{n-1} \leq C^{\prime} r(\phi)$ holds for all $\phi \in\left(0, \phi_{0}\right)$. Since $\delta<t \phi(\delta)$ according to $(2)$, it follows that

$$
\delta^{n-1} \omega_{n-1}<t^{n-1} \phi(\delta)^{n-1} \omega_{n-1} \leq C^{\prime} \frac{\omega_{n-1}}{\omega_{n}} \cdot r(\phi(\delta)) \cdot \omega_{n} t^{n-1}=C^{\prime} \frac{\omega_{n-1}}{\omega_{n}} \cdot \frac{\operatorname{vol}\left(S_{\phi(\delta)}\left(x_{0}, x\right)\right)}{t} .
$$

This estimate holds as long as $\phi(\delta) \in\left(0, \phi_{0}\right)$. Since $t$ is fixed and $t \phi(\delta) / \delta \rightarrow 1$, we can choose $\delta_{0}>0$ suitably such that this condition is satisfied for all $\delta \in\left(0, \delta_{0}\right)$. 


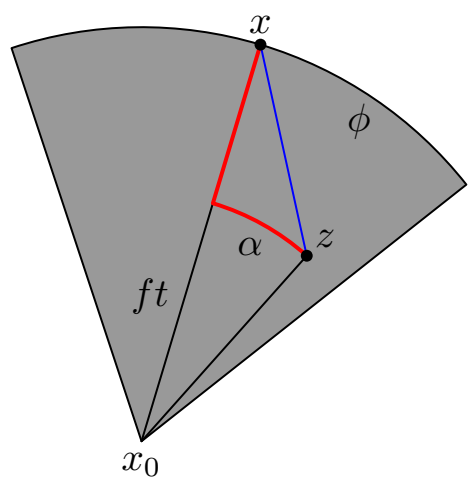

Figure 2: The situation of Lemma 4. The quantity $|x-z|$, shown in blue, is estimated.

\subsection{Sectors Are Disjoint}

Another main ingredient for the proof of Theorem 2 will be Lemma 7 below. Let us now prepare some lemmas that will allow us to prove this result at the end of this subsection. For the first, consider the situation shown in Figure 2. We will derive various estimates of the distance $|x-z|$ highlighted in blue, depending on the angle $\phi$ of the sector as well as the distance $\left|x_{0}-z\right|=f t$. In particular:

Lemma 4. Let $x_{0}, x \in \mathbb{R}^{n}, \phi \in[0, \pi / 2]$ and $z \in \overline{S_{\phi}\left(x_{0}, x\right)}$. Denote $t=\left|x_{0}-x\right|$ and $\left|x_{0}-z\right|=f t$ with some $f \in[0,1]$. Then

$$
t(1-f) \leq|x-z| \leq t(1-f+\phi f) \leq t(1-f+\phi)
$$

and also

$$
|x-z|^{2} \leq t^{2}\left((1-f)^{2}+f \phi^{2}\right)
$$

If $f \leq 1 / 2$, then additionally

$$
|x-z| \leq t\left(1-f+\frac{\phi^{2}}{2}\right) .
$$

Proof. For the first inequality, note that

$$
t=\left|x_{0}-x\right| \leq\left|x_{0}-z\right|+|z-x|=t f+|z-x| .
$$

Furthermore, consider the red path in Figure 2. It is necessarily longer than $|x-z|$. Noting that $\alpha \leq \phi$, we find

$$
|x-z| \leq f t \alpha+(t-f t) \leq t(1-f+f \phi) \leq t(1-f+\phi) .
$$

In fact, using basic trigonometry, one can even deduce the exact relation

$$
|x-z|^{2}=t^{2}\left((1-f \cos \alpha)^{2}+f^{2} \sin ^{2} \alpha\right)=t^{2}\left(1+f^{2}-2 f \cos \alpha\right) .
$$

Since this expression is increasing in $\alpha$, we also get

$$
|x-z|^{2} \leq t^{2}\left(1+f^{2}-2 f \cos \phi\right) \leq t^{2}\left(1+f^{2}-2 f+f \phi^{2}\right)=t^{2}\left((1-f)^{2}+f \phi^{2}\right) .
$$

Here, we have used that $\cos \phi \geq 1-\phi^{2} / 2$ for all $\phi \in \mathbb{R}$. This shows the second inequality.

Finally, let $f \leq 1 / 2$. Note that a series expansion implies

$$
\sqrt{a^{2}+b x^{2}} \leq a+\frac{b}{2 a} x^{2}
$$

for all $a>0$ and $b, x \geq 0$. Applying this with $a=1-f$ and $b=f$ to (8) yields

$$
|x-z| \leq t\left(1-f+\frac{f}{2(1-f)} \phi^{2}\right) .
$$

Since $f \leq 1 / 2$, the coefficient in front of $\phi^{2}$ can be estimated by $1 / 2$. This shows the third inequality. 
Next, we consider two sectors $S_{\phi}\left(x_{0}, x\right)$ and $S_{\phi}\left(y_{0}, y\right)$. The angle is the same for both, and we assume that $\phi=\phi(\delta)$ for some $\delta>0$. As usual, we set $t=\left|x_{0}-x\right|=\left|y_{0}-y\right|$. We are interested in the situation

$$
\overline{B_{\delta}(x)} \cap \overline{B_{\delta}(y)}=\emptyset \text { and } t \leq \min \left(\left|x_{0}-y\right|,\left|y_{0}-x\right|\right)
$$

Our goal is to show that we can make these sectors disjoint by taking appropriate subsets, without reducing their volumes too much in the process. (Actually, we believe that the sectors themselves are already disjoint. We do not yet have a proof for this conjecture, though, and do not need one for our purposes.) Thus, assume $z \in \overline{S_{\phi}\left(x_{0}, x\right)} \cap \overline{S_{\phi}\left(y_{0}, y\right)}$. Let us write $\left|x_{0}-z\right|=f_{1} t$ and $\left|y_{0}-z\right|=f_{2} t$, where $f_{1}, f_{2} \in[0,1]$ as before. Under these assumptions, we can first conclude that $f_{1}$ and $f_{2}$ must be close to each other:

Lemma 5. Consider the situation described above and assume, in particular, that condition (10) is fulfilled. Then $\left|f_{1}-f_{2}\right| \leq \phi$. If furthermore $f_{1}, f_{2} \leq 1 / 2$, then also $\left|f_{1}-f_{2}\right| \leq \phi^{2} / 2$.

Proof. Assume, without loss of generality, $f_{1} \leq f_{2}$. Then (7) implies

$$
t \leq\left|x_{0}-y\right| \leq\left|x_{0}-z\right|+|z-y| \leq f_{1} t+t\left(1-f_{2}+\phi\right)=t\left(1-\left|f_{1}-f_{2}\right|+\phi\right) .
$$

This yields the claimed $\left|f_{1}-f_{2}\right| \leq \phi$. For the case $f_{1}, f_{2} \leq 1 / 2$, the second estimate follows if we use (9) instead of (7) to get an upper bound on $|z-y|$.

While Lemma 5 has already used the second part of condition (10), we now make use of the first part. By knowing that the balls $\overline{B_{\delta}(x)}$ and $\overline{B_{\delta}(y)}$ are disjoint, we can conclude that potential intersection points of the sectors must lie on a line that has a certain minimum angle to the sector's central line:

Lemma 6. We use the notation from above and assume that condition (10) is satisfied. Furthermore, let $0<f_{1}, f_{2} \leq 1 / 2$ and introduce the angle $\alpha$ for a given point $z$ as in Figure 3. I. e.,

$$
(y-z) \cdot\left(x_{0}-z\right)=|z-y|\left|z-x_{0}\right| \cdot \cos (\pi-\alpha) .
$$

Then there exists $\delta_{0}>0$ so that $z \in \overline{S_{\phi(\delta)}\left(x_{0}, x\right)} \cap \overline{S_{\phi(\delta)}\left(y_{0}, y\right)}$ implies $8 \alpha \geq \phi(\delta)$ for all $\delta \in\left(0, \delta_{0}\right)$.

Proof. Since $t \phi(\delta) / \delta \rightarrow 1$ as $\delta \rightarrow 0^{+}$, there exists $\delta_{0}>0$ such that $t \phi(\delta) \leq 2 \delta$ holds for all $\delta \in\left(0, \delta_{0}\right)$. We can choose $\delta_{0}$ so that $\phi(\delta) \leq 1 / 4$ in addition. Now, let $\delta \in\left(0, \delta_{0}\right)$ be given and set $\phi=\phi(\delta)$. Note that, for $n>2$, the four points $x_{0}, z, x$ and $y$ do not necessarily lie in one plane. Thus, let us introduce the point

$$
x^{\prime}=x_{0}+t \frac{z-x_{0}}{\left|z-x_{0}\right|}
$$

on the base of $S_{\phi}\left(x_{0}, x\right)$. (Note that $z \neq x_{0}$ since $f_{1}>0$ is assumed.) With this definition, $x^{\prime}$ lies in the plane defined by $x_{0}, z$ and $y$. In the following, we will consider this plane as depicted in Figure 3. By $\overline{B_{\delta}(x)} \cap \overline{B_{\delta}(y)}=\emptyset$, we know that

$$
\left|x^{\prime}-y\right| \geq|x-y|-\left|x-x^{\prime}\right| \geq 2 \delta-\delta=\delta \geq \frac{t \phi}{2} .
$$

Next, we want to estimate $\left|x^{\prime}-y\right|$ also from above. For this, consider the highlighted path in Figure 3 The length of the circular arc in this path can be estimated via (9) as

$$
l_{1}=\alpha|z-y| \leq t\left(1-f_{2}+\frac{\phi^{2}}{2}\right) \alpha .
$$

The length of the straight part is given by

$$
l_{2}=|| z-x^{\prime}|-| z-y||=\left|t\left(1-f_{1}\right)-\right| z-y|| .
$$

To resolve the absolute value, we have two cases to consider: First, assume $\left|z-x^{\prime}\right| \geq|z-y|$ (as depicted in the sketch). In this case,

$$
l_{2}=t\left(1-f_{1}\right)-|z-y| \leq t\left(1-f_{1}\right)-t\left(1-f_{2}\right)=t\left(f_{2}-f_{1}\right) \leq t\left|f_{2}-f_{1}\right| .
$$




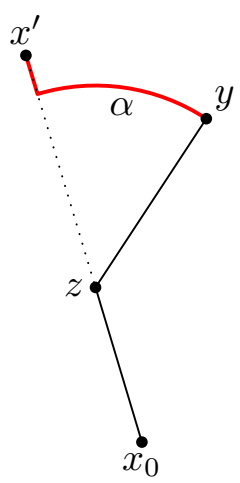

Figure 3: Sketch for the situation considered in the proof of Lemma 6 .

Alternatively, for $\left|z-x^{\prime}\right| \leq|z-y|$, the estimate (9) implies

$$
l_{2}=|z-y|-t\left(1-f_{1}\right) \leq t\left(1-f_{2}+\frac{\phi^{2}}{2}\right)-t\left(1-f_{1}\right)=t\left(f_{1}-f_{2}+\frac{\phi^{2}}{2}\right) \leq t\left(\left|f_{1}-f_{2}\right|+\frac{\phi^{2}}{2}\right) .
$$

Note that the latter upper bound is larger than the one we got in the first case, so that it actually holds for both cases. Since Lemma 5 implies $\left|f_{1}-f_{2}\right| \leq \phi^{2} / 2$, we arrive at the final estimate

$$
\left|x^{\prime}-y\right| \leq l_{1}+l_{2} \leq t\left(1-f_{2}+\frac{\phi^{2}}{2}\right) \alpha+t\left(\left|f_{1}-f_{2}\right|+\frac{\phi^{2}}{2}\right) \leq t\left(\left(1+\frac{\phi^{2}}{2}\right) \alpha+\phi^{2}\right) .
$$

Since $\phi \leq 1 / 4$, also $\phi^{2} / 2 \leq 1$ and $\phi^{2} \leq \phi / 4$. Using (12) and (13) together finally yields

$$
\frac{t \phi}{2} \leq\left|x^{\prime}-y\right| \leq t\left((1+1) \alpha+\frac{\phi}{4}\right)
$$

This can be easily transformed to show the claimed $\phi \leq 8 \alpha$.

Combining the results above, we can finally show that certain well-defined subsets of two such sectors must be disjoint:

Lemma 7. Fix $t>0$. There exist $\delta_{0}>0, a>0$ and $0<\underline{f}<\bar{f} \leq 1$ such that

$$
\overline{S_{a \phi(\delta)}\left(x_{0}, x ; \underline{f}, \bar{f}\right)} \cap \overline{S_{a \phi(\delta)}\left(y_{0}, y ; \underline{f}, \bar{f}\right)}=\emptyset
$$

for all $\delta \in\left(0, \delta_{0}\right)$ and $x_{0}, y_{0}, x, y \in \mathbb{R}^{n}$ with $t=\left|x_{0}-x\right|=\left|y_{0}-y\right|$, whenever condition (10) is satisfied. The constants $a, \underline{f}$ and $\bar{f}$ are explicit and do not depend on any other values.

Proof. Note that there exists $\underline{f} \in(0,1 / 2)$ such that $f(1-f) \geq 1 / 6$ for all $f \in[f, 1 / 2]$. Set $a=1 / 32$ and $\bar{f}=1 / 2$. Assume that there exists $z$ in the intersection (14) Let us use the notation $f_{1}$ and $f_{2}$ as before. Note that $f_{1}, f_{2} \leq 1 / 2$ and $f_{1}\left(1-f_{1}\right) \geq 1 / 6$ by our choice of $f$ and $\bar{f}$. Let $\alpha$ be as in (11). If $\phi$ is small enough, Lemma 6 implies that $8 \alpha \geq \bar{\phi}$. If we set $\phi^{\prime}=a \phi=\bar{\phi} / 32$, this means $\alpha \geq 4 \bar{\phi}^{\prime}$. Recall also that $\left|x_{0}-z\right|=f_{1} t$ by definition, $t \leq\left|x_{0}-y\right|$ by condition $(10)$ and $|z-y| \leq t\left(1-f_{2}+\phi^{\prime 2} / 2\right)$ by Lemma 4. Furthermore, Lemma 5 implies $\left|f_{1}-f_{2}\right| \leq \phi^{\prime 2} / 2$, which, in particular, yields

$$
f_{2} \geq f_{1}-\frac{\phi^{\prime 2}}{2} \Leftrightarrow-f_{2}+\frac{\phi^{\prime 2}}{2} \leq-f_{1}+\phi^{\prime 2} .
$$

Now we are ready to put everything together: Using the law of cosines in the plane containing $x_{0}, z$ and $y$ (see Figure 3 and (11), we get:

$$
\begin{aligned}
t^{2} & \leq\left|x_{0}-y\right|^{2}=\left|x_{0}-z\right|^{2}+|z-y|^{2}-2\left|x_{0}-z\right||z-y| \cos (\pi-\alpha) \\
& =t^{2} f_{1}^{2}+|z-y|^{2}+2 t f_{1}|z-y| \cos \alpha \leq t^{2} f_{1}^{2}+t^{2}\left(1-f_{2}+\frac{\phi^{\prime 2}}{2}\right)^{2}+2 t^{2} f_{1}\left(1-f_{2}+\frac{\phi^{\prime 2}}{2}\right) \cos \alpha \\
& \leq t^{2} f_{1}^{2}+t^{2}\left(1-f_{1}+\phi^{\prime 2}\right)^{2}+2 t^{2} f_{1}\left(1-f_{1}+\phi^{\prime 2}\right) \cos \alpha
\end{aligned}
$$


Dividing by $t^{2}$ and completing the square yields the further estimates:

$$
\begin{aligned}
1 & \leq\left(f_{1}+1-f_{1}+\phi^{\prime 2}\right)^{2}+2 f_{1}\left(1-f_{1}+\phi^{\prime 2}\right)(\cos \alpha-1) \\
& \leq\left(1+\phi^{\prime 2}\right)^{2}-2 f_{1}\left(1-f_{1}\right)(1-\cos \alpha) \leq\left(1+\phi^{\prime 2}\right)^{2}-\frac{2}{6}\left(1-\cos \left(4 \phi^{\prime}\right)\right)
\end{aligned}
$$

With a series expansion, one can see that there exists $\phi_{0}>0$ such that this inequality is wrong for all $\phi^{\prime} \in\left(0, \phi_{0}\right)$. This finishes the proof.

\section{Main Results}

With all the technical preparations of Section 2 in place, we can now proceed to show the main results. As before, let us assume that $\Omega_{0} \subset \mathbb{R}^{n}$ is an open set. We denote its boundary by $\Gamma_{0}=\partial \Omega_{0}$ and introduce $d=d_{\Omega_{0}}$ as the distance function of $\Omega_{0}$. Recall also the definition of $\Omega_{t}$ and $\Gamma_{t}$ from $(1)$.

Lemma 8. For each $x \in \mathbb{R}^{n} \backslash \Omega_{0}$,

$$
d(x)=\inf _{y \in \Gamma_{0}}|x-y|
$$

Furthermore, there exists $x_{0} \in \Gamma_{0}$ with $d(x)=\left|x-x_{0}\right|$.

Proof. See (2.2) on page 337 of 6 for (15)] $\Gamma_{0}$ is closed, and we can clearly restrict the infimum to some bounded subset of $\Gamma_{0}$. Hence, this subset is compact and there exists a minimiser $x_{0}$.

In the following, we are interested in estimating the "surface area" of $\Omega_{t}$ for $t>0$. Before we can do that, let us briefly recall the applicable concepts for defining such a surface area in the first place: For an open set $\Omega \subset \mathbb{R}^{n}$, we denote by $P(\Omega)$ its perimeter as defined, for instance, by Definition 3.35 on page 143 of 1 . (Note that we are always interested in the perimeter relative to the base set $\mathbb{R}^{n}$.) $\Omega$ is said to have finite perimeter or to be a Caccioppoli set if $P(\Omega)<\infty$.

Furthermore, let us introduce also the Hausdorff measure following Definition 2.46 on page 72 of [1:

Definition 2. Let $k \in \mathbb{N}$ and $\Omega \subset \mathbb{R}^{n}$. For $\delta>0$, we define

$$
\mathcal{H}_{\delta}^{k}(\Omega)=\inf \left\{\sum_{i=1}^{\infty}\left(\frac{d_{i}}{2}\right)^{k} \omega_{k}\left|\Omega \subset \bigcup_{i=1}^{\infty} U_{i}, d_{i}=\sup _{x, y \in U_{i}}\right| x-y \mid, d_{i} \leq 2 \delta\right\} .
$$

Here, $\omega_{k}$ denotes the volume of the $k$-dimensional unit ball as in Lemma 1 . The value $d_{i}$ is the diameter of the set $U_{i}$, and it is allowed to be at most $2 \delta$ in order for $\left(U_{i}\right)$ to be an admissible $\delta$-covering of $\Omega$.

Furthermore, the $k$-dimensional Hausdorff measure of $\Omega$ is then given by

$$
\mathcal{H}^{k}(\Omega)=\sup _{\delta>0} \mathcal{H}_{\delta}^{k}(\Omega)=\lim _{\delta \rightarrow 0^{+}} \mathcal{H}_{\delta}^{k}(\Omega) .
$$

Note that we define the Hausdorff measure in such a way that $\mathcal{H}^{n}$ corresponds to the $n$-dimensional Lebesgue measure. (For a proof, see Theorem 2.53 in 1.) This is the reason for including $\omega_{k}$ in the definition. Others (e. g., [14]) do not add this normalisation constant, which results in a notion of $\mathcal{H}^{k}$ that is different from Definition 2 by a constant.

For the case of only one dimension, the situation is simple since sets of finite perimeter in one dimension can be represented (up to a set of measure zero) as the union of a finite number of intervals:

Theorem 1. Let $n=1$ and $\Omega_{0} \subset \mathbb{R}$ be open and bounded. Then $\Gamma_{t}$ is a finite set for each $t>0$ and its cardinality is non-increasing with respect to $t$. Furthermore,

$$
\mathcal{H}^{0}\left(\Gamma_{t}\right) \leq P\left(\Omega_{0}\right)
$$

If $\Omega_{0}$ has finite perimeter and $t$ is sufficiently small, then even equality holds. 
Proof. Let $t>0$ and $x \in \Gamma_{t}$. Lemma 8 implies that there exists $x_{0} \in \Gamma_{0}$ with $\left|x-x_{0}\right|=t$. Assume, without loss of generality, that $x_{0}<x$. It follows that $I_{x}=\left(x_{0}, x\right) \subset d^{-1}((0, t))$. Furthermore, if $y \in \Gamma_{t}$ and $x \neq y$, then $I_{x} \cap I_{y}=\emptyset$. Since $\operatorname{vol}\left(I_{x}\right)=t>0$ for each $x \in \Gamma_{t}$ and $\Omega_{t}$ is bounded, the cardinality of $\Gamma_{t}$ is bounded as $\mathcal{H}^{0}\left(\Gamma_{t}\right) \leq \operatorname{vol}\left(\Omega_{t}\right) / t$ and thus finite. If we have $0<s<t$, the estimate (16) implies that

$$
\mathcal{H}^{0}\left(\Gamma_{t}\right) \leq P\left(\Omega_{s}\right) \leq \mathcal{H}^{0}\left(\Gamma_{s}\right) .
$$

Hence it follows that the cardinality is non-increasing when we have established (16)

For (16), assume that $\Omega_{0}$ has finite perimeter (the situation is trivial otherwise). According to Proposition 3.52 on page 153 of $\left[1\right.$, there exist $p \in \mathbb{N}$ and $p$ disjoint intervals $J_{i}=\left[a_{i}, b_{i}\right]$ such that $\Omega_{0} \subset \bigcup_{i=1}^{p} J_{i}$. These two sets can only differ up to a set of measure zero. Furthermore, $P\left(\Omega_{0}\right)=2 p$. As before, we can associate an interval $I_{x} \subset d^{-1}((0, t))$ to each $x \in \Gamma_{t}$, and all $I_{x}$ are disjoint. If we assume that $I_{x}=\left(x_{0}, x\right)$, then $x_{0}=b_{i}$ for some $1 \leq i \leq p$. Similarly, $x_{0}=a_{i}$ if $I_{x}=\left(x, x_{0}\right)$. This implies (16) since

$$
\mathcal{H}^{0}\left(\Gamma_{t}\right) \leq 2 p=P\left(\Omega_{0}\right)
$$

If we assume an ordering such as

$$
a_{1}<b_{1}<a_{2}<b_{2}<\cdots<a_{p}<b_{p}
$$

and denote by

$$
L=\inf _{i=1, \ldots, p-1}\left(a_{i+1}-b_{i}\right)>0
$$

the minimal distance between the intervals $J_{i}$, then equality holds with $\mathcal{H}^{0}\left(\Gamma_{t}\right)=2 p$ for $t<L / 2$.

\subsection{A Bound on the Hausdorff Measure}

Intuitively, $\Omega_{t}$ is constructed from $\Omega_{0}$ by adding a "layer" of thickness $t$ onto $\Gamma_{0}$. Following this picture, one can imagine that the volume of this layer should roughly equal $t$ times the surface area (i. e., perimeter) of either $\Omega_{0}$ or $\Omega_{t}$. This argument can be made rigorous by estimating the volume in terms of $P\left(\Omega_{0}\right)$ and then $\mathcal{H}^{n-1}\left(\Gamma_{t}\right)$ in terms of the volume. The former will be done in Subsection 3.2. We will show the latter as our first main result in this subsection. This is, somehow, an inverse isoperimetric inequality. Of course, in the general situation no inverse to the classical isoperimetric inequality (see section 5.6 .2 of [9]) holds. In our case, however, it works because the considered volume is not allowed to be "arbitrarily thin".

Definition 3. For $\Omega_{0}$, we define the newly created volume to be

$$
U_{t}=\left(\bigcup_{x_{0} \in \Gamma_{0}} B_{t}\left(x_{0}\right)\right) \backslash\left(\Gamma_{0} \cup \Omega_{0}\right)=\left\{x \in \mathbb{R}^{n} \mid 0<d(x)<t\right\} .
$$

We can now state and prove the first main result:

Theorem 2. There exists a dimensional constant $C$ such that

$$
P\left(\Omega_{t}\right) \leq \mathcal{H}^{n-1}\left(\Gamma_{t}\right) \leq C \frac{\operatorname{vol}\left(U_{t}\right)}{t}
$$

holds for all $t>0$.

Proof. The first inequality is a well-known fact about the relation between perimeter and the Hausdorff measure. See, for instance, Proposition 3.62 on page 159 of 1 . We will now show the second inequality. For this, let $\delta_{0}$ be the bound from Lemma 7 and $\delta \in\left(0, \delta_{0}\right)$ be given. Then clearly $\Gamma_{t} \subset \cup_{x \in \Gamma_{t}} \overline{B_{\delta}(x)}$. According to Vitali's covering theorem (see Theorem 1 on page 27 of [9]), there exists a countable subset $X \subset \Gamma_{t}$ such that

$$
\Gamma_{t} \subset \bigcup_{x \in X} \overline{B_{5 \delta}(x)}
$$

and all $\overline{B_{\delta}(x)}$ are disjoint for $x \in X$. Note that $X$ is, in fact, finite if $\Omega_{0}$ is bounded. 
For each $x \in \Gamma_{t}$, there exists a corresponding $x_{0} \in \Gamma_{0}$ with $\left|x-x_{0}\right|=t$ according to Lemma 8 Furthermore, $t \leq\left|y-y_{0}\right|$ for all $y \in \Gamma_{t}$ and $y_{0} \in \Gamma_{0}$. Based on Lemma 7 , define

$$
S_{x}=\overline{S_{a \phi(\delta)}\left(x_{0}, x ; \underline{f}, \bar{f}\right)}
$$

for all $x \in X$. Note that the condition (10) is satisfied for each pair $\left(S_{x}, S_{y}\right)$ with $x, y \in X$, so that all $S_{x}$ and $S_{y}$ with $x \neq y$ are disjoint by Lemma 7. Also note that $S_{x} \cap\left(\Gamma_{0} \cup \Omega_{0}\right)=\emptyset$ as long as $\delta$ is small enough: For $z \in S_{x}$, we know from (7) that

$$
|x-z| \leq t(1-f+a \phi(\delta))<t(1-f+\phi(\delta)),
$$

where $f=\left|x_{0}-z\right| / t \geq \underline{f}$. Thus, $|x-z|<t$ if $\phi(\delta) \leq \underline{f}$. Since $d(x)=t$, this implies $z \notin \Gamma_{0} \cup \Omega_{0}$. Thus, we also find that each $S_{x}$ is contained in the newly created volume and get

$$
\sum_{x \in X} \operatorname{vol}\left(S_{\phi(\delta)}\left(x_{0}, x\right)\right) \leq C^{\prime} \sum_{x \in X} \operatorname{vol}\left(S_{x}\right)=C^{\prime} \cdot \operatorname{vol}\left(\bigcup_{x \in X} S_{x}\right) \leq C^{\prime} \cdot \operatorname{vol}\left(U_{t}\right) .
$$

As long as $\delta$ is small enough, a suitable $C^{\prime}$ exists based on an estimate similar to (6)

Since the enlarged balls provide a particular $5 \delta$-covering of $\Gamma_{t}$, we know that

$$
\mathcal{H}_{5 \delta}^{n-1}\left(\Gamma_{t}\right) \leq \sum_{x \in X}(5 \delta)^{n-1} \omega_{n-1} \leq 5^{n-1} \frac{C^{\prime \prime}}{t} \sum_{x \in X} \operatorname{vol}\left(S_{\phi(\delta)}\left(x_{0}, x\right)\right) .
$$

The last estimate and the constant $C^{\prime \prime}$ come from Lemma 3 . Together with (17) this yields

$$
\mathcal{H}_{5 \delta}^{n-1}\left(\Gamma_{t}\right) \leq 5^{n-1} C^{\prime} C^{\prime \prime} \cdot \frac{\operatorname{vol}\left(U_{t}\right)}{t} .
$$

Since the bound on the right-hand side does not depend on $\delta$ any more, this implies the claim in the limit $\delta \rightarrow 0^{+}$.

Having this first result, we can already show that all evolved sets $\Omega_{t}$ must be Caccioppoli sets:

Corollary 1. Let $\Omega_{0}$ be bounded. Then $\Omega_{t}$ has finite perimeter for $t>0$.

Proof. From the boundedness of $\Omega_{0}$, we can directly conclude that also $\Omega_{t}$ and $U_{t}$ are bounded sets for any fixed $t$. Thus, vol $\left(U_{t}\right)<\infty$ and Theorem 2 implies that $\mathcal{H}^{n-1}\left(\Gamma_{t}\right)$ is finite for each $t$. It follows now again from Proposition 3.62 on page 159 of 1$]$ that $\Omega_{t}$ is a set of finite perimeter.

Take note that the actual bound we get from Corollary 1 diverges like $1 / t$ for $t \rightarrow 0^{+}$. It will be the focus of the next subsection (in particular, Corollary 2) to show a uniform bound as $t \rightarrow 0^{+}$under additional assumptions. Section 4 (in particular, Theorem 5 will be devoted to a construction that shows that the rate $1 / t$ is (almost) sharp if these additional assumptions are not fulfilled.

\subsection{Uniform Bounds}

As we have seen above in Theorem 2 , the quantity vol $\left(U_{t}\right) / t$ is crucial as it gives an upper bound on the evolved sets' perimeters. Particularly interesting is the limit $t \rightarrow 0^{+}$. As our second main result below, we can show that there exists a uniform upper bound for $t \rightarrow 0^{+}$as long as a uniform density condition holds for the initial set $\Omega_{0}$. This condition prevents arbitrarily sharp corners and cusps. To be precise:

Definition 4. Let $A \subset \Gamma_{0}, c \in(0,1)$ and $t_{0}>0$. We say that $\Omega_{0}$ has $\left(t_{0}, c\right)$-uniform lower density on $A$ if the estimate

$$
0<c \leq \frac{\operatorname{vol}\left(B_{t}(x) \cap \Omega_{0}\right)}{\operatorname{vol}\left(B_{t}(x)\right)}
$$

holds for all $t \in\left(0, t_{0}\right)$ and $x \in A$.

Similarly, $\Omega_{0}$ is said to have $\left(t_{0}, c\right)$-uniform upper density on $A$ if

$$
\frac{\operatorname{vol}\left(B_{t}(x) \cap \Omega_{0}\right)}{\operatorname{vol}\left(B_{t}(x)\right)} \leq 1-c<1 .
$$

When both conditions are satisfied together, $\Omega_{0}$ simply has $\left(t_{0}, c\right)$-uniform density on $A$. 
For fixed $x$ and in the limit $t \rightarrow 0^{+}$, the expression in $(18)$ and (19) gives the density of $\Omega_{0}$ at $x$. See page 158 of $[1$ for some known results about this quantity. In particular, let $\mathcal{F} \Omega$ denote the reduced boundary of an open set $\Omega$. (Roughly speaking, this is the set of all boundary points where a measure-theoretic variant of the normal vector to the boundary can be defined. See Definition 3.54 on page 154 of 1 .) Then $\Omega$ has density $1 / 2$ at all points in $\mathcal{F} \Omega$. This is, for instance, also true in the example constructed in Section 4. Hence, note that uniformity of the estimates is really crucial for our purposes in the following. The relation between this concept of uniform density and other, more established geometric properties will be discussed in more detail in Subsection 3.3.

For our estimate of vol $\left(U_{t}\right)$, we need to somehow get an upper bound on $t$ in terms of the perimeter of $\Omega_{0}$. For a classical result in this direction, see (3.54) on page 156 of [1]. Unfortunately, this estimate is local in nature and not uniform over the whole boundary of $\Omega_{0}$. Note, however, that $(18)$ and $(19)$ together are equivalent to

$$
c \leq \frac{\min \left(\operatorname{vol}\left(B_{t}(x) \cap \Omega_{0}\right), \operatorname{vol}\left(B_{t}(x) \backslash \Omega_{0}\right)\right)}{\operatorname{vol}\left(B_{t}(x)\right)} .
$$

This relation can be combined with the relative isoperimetric inequality (see, for instance, Theorem 2 on page 190 of [9]) to get the uniform estimate that we need:

Lemma 9. Let $\Omega_{0}$ have $\left(t_{0}, c\right)$-uniform density on $A$. Then there exists a dimensional constant $C$ such that

$$
t^{n-1} \leq C\left(\frac{1}{c}\right)^{\frac{n-1}{n}} \mathcal{H}^{n-1}\left(B_{t}(x) \cap \mathcal{F} \Omega_{0}\right)
$$

for all $x \in A$ and $t \in\left(0, t_{0}\right)$.

Proof. Since we assume uniform density, (20) implies that

$$
c \cdot \operatorname{vol}\left(B_{t}(x)\right)=c \cdot \omega_{n} t^{n} \leq \min \left(\operatorname{vol}\left(B_{t}(x) \cap \Omega_{0}\right), \operatorname{vol}\left(B_{t}(x) \backslash \Omega_{0}\right)\right)
$$

for all $t \in\left(0, t_{0}\right)$. If we also apply the relative isoperimetric inequality, we get

$$
t^{n-1} \leq C\left(\frac{1}{c}\right)^{\frac{n-1}{n}} P\left(\Omega_{0} ; B_{t}(x)\right)
$$

for some dimensional constant $C$. This implies the result together with the well-known relation between perimeter and $\mathcal{H}^{n-1}$ (Theorem 3.59 on page 157 of $[1]$ ).

So far, we have assumed uniform density of $\Omega_{0}$. It will turn out, however, that it is enough to require only uniform lower density. Uniform upper density is provided automatically if we choose the subset $A \subset \Gamma_{0}$ in the right way:

Definition 5. We say that $x_{0} \in \Gamma_{0}$ is backwards reachable for time $t>0$ if there exists $x \in \mathbb{R}^{n}$ with

$$
t \leq\left|x_{0}-x\right|=d(x)
$$

The set of all backwards reachable points for time $t$ is denoted by $R_{t}$.

See Figure 4a for an illustration of the set $R_{t}$ : The point $x_{0} \in R_{t}$ is shown together with a possible $x \in \Gamma_{t}$ that fulfils (21) Note that only the red part of $\Gamma_{0}$ is backwards reachable. Thus, we see that $R_{t}$ is actually more regular than $\Gamma_{0}$ itself. In particular, $\Omega_{0}$ has always uniform upper density on $R_{t}$. To understand why this must be the case, take a look at Figure $4 \mathrm{~b}$. Whenever $x_{0}$ and $x$ are as indicated, the ball $B_{t}(x)$ must be disjoint to $\Gamma_{0} \cup \Omega_{0}$ since otherwise $d(x)<t$ would be the case. Thus, the volume of $B_{t}(x) \cap B_{t}\left(x_{0}\right)$ can never be part of $\Omega_{0}$, which implies an upper bound for the density of $\Omega_{0}$ at $x_{0}$. (For the shown situation, the density is actually $1 / 2$. The maximal possible density would be achieved if also the light grey area were part of $\Omega_{0}$.) Let us formalise this argument:

Lemma 10. Let $t>0$ and $R_{t}$ be the backwards reachable set for time $t$. Then $\Omega_{0}$ has $(t, c)$-uniform upper density on $R_{t}$, where $c$ is a dimensional constant. 


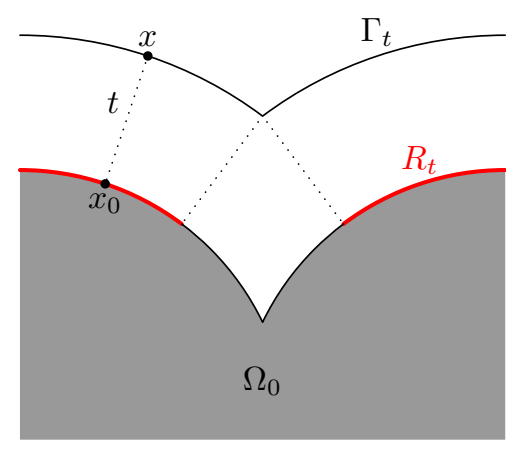

(a) The set $R_{t}$ of Definition 5

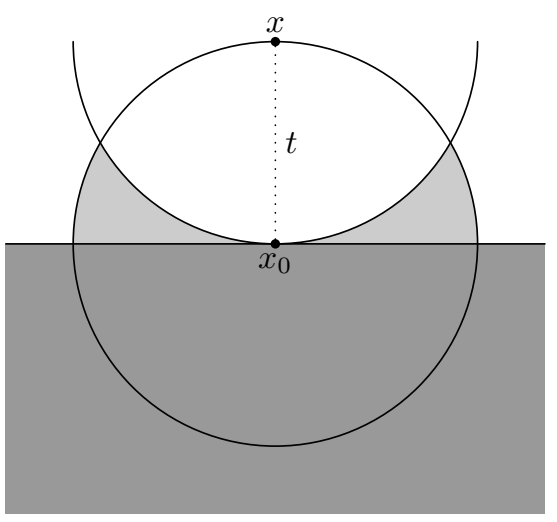

(b) The main argument in the proof of Lemma 10

Figure 4: The backwards reachable set and its regularity with respect to uniform upper density. The dark grey region is $\Omega_{0}$. The point $x_{0}$ is on $R_{t} \subset \Gamma_{0}$, with $x \in \Gamma_{t}$ such that (21) holds.

Proof. Let $e \in \mathbb{R}^{n}$ be arbitrary with $|e|=1$. We define

$$
0<c=\frac{\operatorname{vol}\left(B_{1}(0) \cap B_{1}(e)\right)}{\operatorname{vol}\left(B_{1}(0)\right)}<1 .
$$

Now choose $x_{0} \in R_{t}$ and $\tau \leq t$. We have to show that (19) holds for $B_{\tau}\left(x_{0}\right)$ with the defined $c$. By Definition 5. there exists $x \in \mathbb{R}^{n}$ such that $\tau \leq t \leq\left|x_{0}-x\right|=d(x)$. We can assume, without loss of generality, that $\left|x_{0}-x\right|=\tau$. Considering Figure $4 \mathrm{~b}$, this implies $B_{\tau}(x) \cap \Omega_{0}=\emptyset$. Hence:

$$
\frac{\operatorname{vol}\left(B_{\tau}\left(x_{0}\right) \cap \Omega_{0}\right)}{\operatorname{vol}\left(B_{\tau}\left(x_{0}\right)\right)}=1-\frac{\operatorname{vol}\left(B_{\tau}\left(x_{0}\right) \backslash \Omega_{0}\right)}{\operatorname{vol}\left(B_{\tau}\left(x_{0}\right)\right)} \leq 1-\frac{\operatorname{vol}\left(B_{\tau}\left(x_{0}\right) \cap B_{\tau}(x)\right)}{\operatorname{vol}\left(B_{\tau}\left(x_{0}\right)\right)}=1-c
$$

Another important observation is that the backwards reachable set is already sufficient for the construction of the newly created volume $U_{t}$. This allows us to restrict ourselves to the more regular $R_{t}$ instead of $\Gamma_{0}$ itself later on.

Lemma 11. For $0<s<t, R_{t} \subset R_{s}$. Furthermore,

$$
U_{t} \backslash U_{s} \subset \bigcup_{x_{0} \in R_{s}} B_{t}\left(x_{0}\right) .
$$

Proof. The inclusion $R_{t} \subset R_{s}$ is immediately clear from Definition 5. Pick $x \in U_{t} \backslash U_{s}$ arbitrarily. By Lemma 8 we can find $x_{0} \in \Gamma_{0}$ with $d(x)=\left|x_{0}-x\right|$. Moreover, $x \notin U_{s}$ implies that $d(x) \geq s$, so that $x_{0} \in R_{s}$. Similarly, $x \in U_{t}$ yields $d(x)<t$ and thus $x \in B_{t}\left(x_{0}\right)$.

With this result, all preparations are in place and we can proceed to the actual estimate of vol $\left(U_{t}\right)$. This is done in two steps: First, we estimate vol $\left(U_{2 t} \backslash U_{t}\right)$. The regularity of the backwards reachable set with respect to uniform upper density of $\Omega_{0}$ can be used for this situation. Afterwards, we build the union of a sequence of such strips in order to get vol $\left(U_{t}\right)$ itself.

Lemma 12. Assume that $\Omega_{0}$ has $\left(t_{0}, c\right)$-uniform lower density on $\Gamma_{0}$. Then there exists a dimensional constant $C$ such that

$$
\operatorname{vol}\left(U_{2 t} \backslash U_{t}\right) \leq C\left(1+\frac{1}{c}\right)^{\frac{n-1}{n}} t \cdot P\left(\Omega_{0}\right)
$$

holds for all $t \in\left(0, t_{0}\right)$. 
Proof. According to Lemma 10, we know that $\Omega_{0}$ has $\left(t, c^{\prime}\right)$-uniform upper density on $R_{t}$ with some dimensional $c^{\prime}$. Since it has uniform lower density per assumption, it has $\left(t, c^{\prime \prime}\right)$-uniform density (both upper and lower) with $c^{\prime \prime}=\min \left(c, c^{\prime}\right)$. Furthermore, note that

$$
\frac{1}{c^{\prime \prime}}=\frac{1}{\min \left(c, c^{\prime}\right)} \leq \frac{1}{c}+\frac{1}{c^{\prime}} .
$$

Thus Lemma 9 implies that

$$
t^{n} \leq C^{\prime}\left(\frac{1}{c}+\frac{1}{c^{\prime}}\right)^{\frac{n-1}{n}} t \cdot \mathcal{H}^{n-1}\left(B_{t}\left(x_{0}\right) \cap \mathcal{F} \Omega_{0}\right)
$$

for all $x_{0} \in R_{t}$ with some dimensional $C^{\prime}$. Taking it even further, this yields also

$$
\operatorname{vol}\left(\overline{B_{10 t}\left(x_{0}\right)}\right) \leq C\left(1+\frac{1}{c}\right)^{\frac{n-1}{n}} t \cdot \mathcal{H}^{n-1}\left(B_{t}\left(x_{0}\right) \cap \mathcal{F} \Omega_{0}\right)
$$

for yet another dimensional constant $C$.

Making use of Lemma 11, we know that

$$
U_{2 t} \backslash U_{t} \subset \bigcup_{x_{0} \in R_{t}} B_{2 t}\left(x_{0}\right) .
$$

With Vitali's covering theorem (see, again, Theorem 1 on page 27 of 9 ), we can construct $X \subset R_{t}$ at most countable such that the sets $B_{2 t}\left(x_{0}\right)$ are disjoint for $x_{0} \in X$, but still

$$
U_{2 t} \backslash U_{t} \subset \bigcup_{x_{0} \in X} \overline{B_{10 t}\left(x_{0}\right)} .
$$

Taking the measure on both sides of this inclusion and using (22), we finally find

$$
\begin{aligned}
\operatorname{vol}\left(U_{2 t} \backslash U_{t}\right) & \leq \sum_{x_{0} \in X} \operatorname{vol}\left(\overline{B_{10 t}\left(x_{0}\right)}\right) \leq C\left(1+\frac{1}{c}\right)^{\frac{n-1}{n}} t \cdot \sum_{x_{0} \in X} \mathcal{H}^{n-1}\left(B_{t}\left(x_{0}\right) \cap \mathcal{F} \Omega_{0}\right) \\
& \leq C\left(1+\frac{1}{c}\right)^{\frac{n-1}{n}} t \cdot \mathcal{H}^{n-1}\left(\mathcal{F} \Omega_{0}\right)=C\left(1+\frac{1}{c}\right)^{\frac{n-1}{n}} t \cdot P\left(\Omega_{0}\right) .
\end{aligned}
$$

The simplification of the sum is justified because all sets $B_{t}\left(x_{0}\right)$ are disjoint.

Theorem 3. Let $\Omega_{0}$ have $\left(t_{0}, c\right)$-uniform lower density on $\Gamma_{0}$. Then

$$
\frac{\operatorname{vol}\left(U_{t}\right)}{t} \leq C\left(1+\frac{1}{c}\right)^{\frac{n-1}{n}} P\left(\Omega_{0}\right)
$$

for all $t \in\left(0, t_{0}\right)$ and a dimensional constant $C$. In particular,

$$
\limsup _{t \rightarrow 0^{+}} \frac{\operatorname{vol}\left(U_{t}\right)}{t} \leq C\left(1+\frac{1}{c}\right)^{\frac{n-1}{n}} P\left(\Omega_{0}\right) .
$$

Proof. Let $t \in\left(0, t_{0}\right)$ be given. Then the disjoint telescopic decomposition

$$
U_{t}=\left(U_{t} \backslash U_{t / 2}\right) \cup\left(U_{t / 2} \backslash U_{t / 4}\right) \cup \cdots=\bigcup_{n=1}^{\infty}\left(U_{2 t / 2^{n}} \backslash U_{t / 2^{n}}\right)
$$

holds. Together with Lemma 12 this yields

$$
\operatorname{vol}\left(U_{t}\right)=\sum_{n=1}^{\infty} \operatorname{vol}\left(U_{2 t / 2^{n}} \backslash U_{t / 2^{n}}\right) \leq C\left(1+\frac{1}{c}\right)^{\frac{n-1}{n}} P\left(\Omega_{0}\right) \cdot \sum_{n=1}^{\infty} \frac{t}{2^{n}}=C\left(1+\frac{1}{c}\right)^{\frac{n-1}{n}} t \cdot P\left(\Omega_{0}\right) .
$$

This finishes the proof. 
When we combine Theorem 3 with Theorem 2 , we finally get a uniform bound for $\mathcal{H}^{n-1}\left(\Gamma_{t}\right)$ :

Corollary 2. Assume that $\Omega_{0}$ has $\left(t_{0}, c\right)$-uniform lower density on $\Gamma_{0}$ and that $\Omega_{0}$ is bounded. In particular, let $\Omega_{0} \subset B_{R}(0)$ for some $R>0$. Then

$$
\mathcal{H}^{n-1}\left(\Gamma_{t}\right) \leq C \cdot\left(1+P\left(\Omega_{0}\right)+t^{n-1}\right)
$$

for all $t \geq 0$. The constant $C$ depends only on $n, t_{0}, c$ and $R$ but no other properties of $\Omega_{0}$.

Proof. Note that the situation is clear for $t=0$ as long as we choose $C \geq 1$. From Theorem 3 , we know that $\operatorname{vol}\left(U_{t}\right) / t \leq C^{\prime} P\left(\Omega_{0}\right)$ for all $t \in\left(0, t_{0}\right)$. Furthermore, since $\Omega_{0} \subset B_{R}(0)$, note that $\Omega_{t} \subset B_{R+t}(0)$. Thus, for $t \geq t_{0}$,

$$
\operatorname{vol}\left(U_{t}\right) \leq \omega_{n}(R+t)^{n} \leq C^{\prime \prime}\left(1+t^{n}\right) \Rightarrow \frac{\operatorname{vol}\left(U_{t}\right)}{t} \leq C^{\prime \prime}\left(\frac{1}{t}+t^{n-1}\right) \leq \frac{C^{\prime \prime}}{t_{0}}\left(1+t^{n-1}\right) .
$$

The claim now follows from Theorem 2, if we combine both estimates for vol $\left(U_{t}\right) / t$.

\subsection{Geometric Regularity Properties in the Literature}

The main ingredient for the results in the previous Subsection 3.2 is a particular geometric property of the initial set $\Omega_{0}$, namely uniform density from Definition 4 This notion is, as far as we know, new and specifically tailored towards our results. Thus, it makes sense to put it into perspective with similar geometric properties that are more established in the literature. In particular, a variety of so-called (uniform) segment and cone properties is often used to characterise geometric regularity of sets. For a thorough introduction, see section 2.6 of $[6]$.

Since uniformity plays an important role for the derivations in Subsection 3.2, it makes only sense to consider the uniform variants of those segment properties. (All non-uniform properties are fulfilled by the example developed in Section 4, since it is constructed only from circles.) Furthermore, the uniform (fat) segment property alone also provides very little regularity. For instance, a cusp satisfies it while it clearly does not have uniform lower density. Thus, let us focus on the uniform cone property. For convenience, we recall its definition from Definition 6.3 on page 115 of [6]:

Definition 6. For $x_{0}, x \in \mathbb{R}^{n}$ and $\phi \in[0, \pi / 2]$, define the open cone

$$
C_{\phi}\left(x_{0}, x\right)=\left\{y \in \mathbb{R}^{n}|| x_{0}-y|| x_{0}-x\left|\cdot \cos \phi<\left(y-x_{0}\right) \cdot\left(x-x_{0}\right)<\right| x_{0}-\left.x\right|^{2}\right\} .
$$

This is similar to the definition of $S_{\phi}\left(x_{0}, x\right)$ in Definition 1, but it describes a cone with flat base (i. e., without a spherical cap).

Now, let $\Omega \subset \mathbb{R}^{n}$ be open. We say that $\Omega$ satisfies the uniform cone property if there exist $t>0$, $\phi \in(0, \pi / 2)$ and $\rho>0$ such that for all $x_{0} \in \partial \Omega$ there is $x \in \mathbb{R}^{n}$ with $\left|x_{0}-x\right|=t$ and

$$
x+d \in \bar{\Omega} \Rightarrow C_{\phi}\left(x_{0}+d, x+d\right) \subset \Omega
$$

for all $d \in B_{\rho}(0)$.

Since the uniform cone property ensures for each boundary point the existence of a cone that is entirely contained in $\Omega$, we can use this cone's volume as a lower bound on the density of $\Omega$. Thus, the uniform cone property is a stronger condition than uniform lower density:

Theorem 4. Let $\Omega \subset \mathbb{R}^{n}$ satisfy the uniform cone property with $t$ and $\phi$ as in Definition 6 . Then $\Omega$ has $(t, r(\phi))$-uniform lower density on $\partial \Omega$. Similarly, if $\mathbb{R}^{n} \backslash \bar{\Omega}$ has the uniform cone property with these constants, then $\Omega$ has $(t, r(\phi))$-uniform upper density.

Proof. Let $x_{0} \in \partial \Omega$ be given. According to Definition 6 , there exists $x \in \mathbb{R}^{n}$ with $\left|x_{0}-x\right|=t$ such that $C_{\phi}\left(x_{0}, x\right) \subset \Omega$. Note that $S_{\phi}\left(x_{0}, x\right) \subset C_{\phi}\left(x_{0}, x\right)$ since

$$
\left|x_{0}-y\right|<\left|x_{0}-x\right| \Rightarrow\left(y-x_{0}\right) \cdot\left(x-x_{0}\right) \leq\left|y-x_{0}\right| \cdot\left|x-x_{0}\right|<\left|x_{0}-x\right|^{2} .
$$


Thus, for each $\tau \in(0, t)$, clearly

$$
B_{\tau}\left(x_{0}\right) \cap S_{\phi}\left(x_{0}, x\right) \subset B_{\tau}\left(x_{0}\right) \cap \Omega
$$

Hence, we can estimate

$$
\operatorname{vol}\left(B_{\tau}\left(x_{0}\right) \cap \Omega\right) \geq \operatorname{vol}\left(B_{\tau}\left(x_{0}\right) \cap S_{\phi}\left(x_{0}, x\right)\right)=r(\phi) \cdot \operatorname{vol}\left(B_{\tau}\left(x_{0}\right)\right)
$$

based on (3). This shows the claim. The proof for uniform upper density works analogously.

Another concept related to our definition of uniform lower density are sets with finite density perimeter as defined in [3] and section 3.1 of 7 :

Definition 7. Let $\Omega \subset \mathbb{R}^{n}$ be open and $h>0$. The $h$-density perimeter of $\Omega$ is defined as

$$
P_{h}(\Omega)=\sup _{0<\epsilon<h} \frac{\operatorname{vol}\left(V_{\epsilon}(\partial \Omega)\right)}{2 \epsilon}
$$

where $V_{\epsilon}(\partial \Omega)$ is the $\epsilon$-envelope of $\partial \Omega$ :

$$
V_{\epsilon}(\partial \Omega)=\bigcup_{x \in \partial \Omega} B_{\epsilon}(x)=\left\{x \in \mathbb{R}^{n} \mid d_{\partial \Omega}(x)<\epsilon\right\}
$$

If $P_{h}(\Omega)$ is finite, we call $\Omega$ a set of finite $h$-density perimeter.

This can be interpreted as a relaxation of the $(n-1)$-dimensional Minkowski content (see, for instance, 3.2.27 in [10]). To be precise, the Minkowski content results if the supremum in (24) is replaced by the limit $\epsilon \rightarrow 0^{+}$. It is easy to see that $V_{\epsilon}\left(\Omega_{0}\right)$ is related to the newly created volume $U_{\epsilon}$ defined in Definition 3 . The set $U_{\epsilon}$ is the part of $V_{\epsilon}\left(\Omega_{0}\right)$ which is outside of $\Gamma_{0} \cup \Omega_{0}$. Hence, an argument similar to the proof of Theorem 3 can be applied to show that uniform density implies finite density perimeter.

\section{Diverging Boundary Length}

In Corollary 1, we have established an upper bound for $P\left(\Omega_{t}\right)$ that behaves like $1 / t$ in the limit $t \rightarrow 0^{+}$. If $\Omega_{0}$ has additional regularity (in particular, uniform lower density), we even get a uniform bound by Corollary 2. We will now show by means of an example that these results cannot be further improved:

Example 1. Consider $D=[0,2] \times[0,1] \subset \mathbb{R}^{2}$ as hold-all domain. For $k=0,1, \ldots$, define

$$
l_{k}=4^{-k}, \quad r_{k}=\frac{\left(l_{k}\right)^{2}}{4}=\frac{1}{4} \cdot 16^{-k}, \quad N_{k}=\frac{2^{-k}}{\left(l_{k}\right)^{2}}=8^{k} .
$$

Based on these definitions, we define $\Omega_{0}$ as infinite union of balls as depicted in Figure 5 . Specifically, $\Omega_{0}$ is constructed by splitting $D$ first into a sequence of vertical strips with widths $2^{-k}$. Each strip is then further divided into squares of size $l_{k} \times l_{k}$. Into each such square, we put a ball with radius $r_{k}$. For each $k$, there is a total of $N_{k}$ such squares and balls.

Each ball at level $k$ has perimeter $2 \pi r_{k}$, so that the total perimeter of $\Omega_{0}$ is given as

$$
P\left(\Omega_{0}\right)=\sum_{k=0}^{\infty} N_{k} \cdot 2 \pi r_{k}=\frac{\pi}{2} \sum_{k=0}^{\infty}\left(\frac{8}{16}\right)^{k}=\pi .
$$

Thus, $\Omega_{0}$ is a bounded set of finite perimeter. It is also clear that it has a smooth boundary, since it consists entirely of balls. Note, however, that it does not have uniform lower density (nor a uniformly bounded curvature), since the balls' radii become arbitrarily small. For the time evolution of $\Omega_{0}$, note that each circle grows outwards and is a circle of radius $r_{k}+t$ at time $t$. This works as long as $t$ is small enough, so that the circle does not yet hit another growing circle. If we let $t_{k}$ be the time at which circles at level $k$ hit their enclosing square, we find that

$$
t_{k}=\frac{l_{k}}{2}-r_{k}=\frac{1}{2} \cdot 4^{-k}-\frac{1}{4} \cdot 16^{-k}=\frac{1}{2} \cdot 4^{-k}\left(1-\frac{1}{2} \cdot 4^{-k}\right) \geq \frac{1}{4} \cdot 4^{-k} .
$$




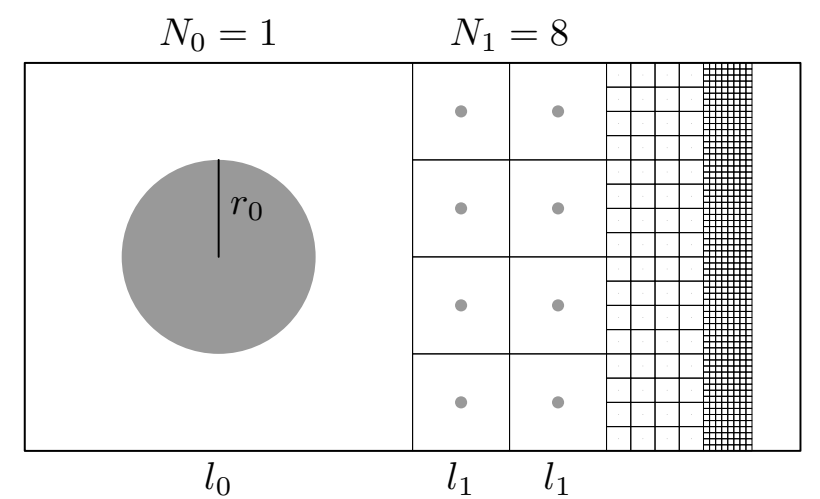

Figure 5: The notation and initial set $\Omega_{0}$ used in Example 1. $\Omega_{0}$ consists of the union of all grey balls.

The other way round, this means that for times $t<t_{k}$, all circles up to (and including) level $k$ have certainly not touched any others. Let $t>0$ be given, and $m$ such that $t_{m+1} \leq t<t_{m}$. If we use only circles up to level $m$ to estimate the perimeter of $\Omega_{t}$, this yields

$$
P\left(\Omega_{t}\right) \geq \sum_{k=0}^{m} N_{k} \cdot 2 \pi\left(r_{k}+t\right) \geq 2 \pi \sum_{k=0}^{m} N_{k} t \geq 2 \pi t_{m+1} \sum_{k=0}^{m} 8^{k} \geq \frac{\pi}{2} \frac{1}{4^{m+1}} \frac{8^{m+1}-1}{7} \geq \frac{\pi}{14}\left(2^{m+1}-1\right)
$$

Note that we can see already here that this expression is unbounded for $t \rightarrow 0^{+}$, since this limit corresponds to $m \rightarrow \infty$. To get a more precise estimate, we can rewrite (25) to get

$$
4^{m} \geq \frac{1}{4 t_{m}} \Leftrightarrow 2^{m} \geq \frac{1}{2 \sqrt{t_{m}}} \Rightarrow 2^{m+1} \geq \frac{1}{2 \sqrt{t_{m+1}}} \geq \frac{1}{2 \sqrt{t}} .
$$

Combining this result with (26) finally gives

$$
P\left(\Omega_{t}\right) \geq \frac{\pi}{14}\left(\frac{1}{2 \sqrt{t}}-1\right)
$$

which diverges like $1 / \sqrt{t}$ as $t \rightarrow 0^{+}$and certainly becomes unbounded.

If one considers the calculation in Example 1 carefully, one can see that the base number in the definition of $l_{k}$ (4 in the example) influences only the constant in front of the final estimate as long as it is larger than 2 . The exponent $1 / 2$ determining the rate to be $1 / \sqrt{t}$ comes from the fact that each level of balls gets assigned only half the area that was assigned to the previous level. We can increase this fraction as long as it is less than 1 if we still want to get a bounded set as result. This line of thought can be extended to the following result:

Theorem 5. Let $n \geq 2$ and $0<s<1$ be given. There exists a Caccioppoli set $\Omega_{0} \subset \mathbb{R}^{n}$ bounded and with smooth boundary, such that

$$
P\left(\Omega_{t}\right) \geq \frac{C}{t^{s}}
$$

for some constant $C$ and $t>0$ small enough. In particular, this rate of divergence holds in the limit $t \rightarrow 0^{+}$.

Proof. We replicate the construction of Example 1. For the desired result, choose some $\alpha>1$ and set

$$
f=\alpha^{s-1} \in(0,1) .
$$

Note that $f \alpha^{n}>f \alpha=\alpha^{s}>1$. We define

$$
l_{k}=\alpha^{-k}, \quad r_{k}=\frac{\left(l_{k}\right)^{n}}{4}=\frac{\alpha^{-k n}}{4}, \quad N_{k}=\left\lceil f^{k} \alpha^{n k}\right\rceil .
$$


This yields to a total volume of all $\left(l_{k}\right)^{n}$-cubes of

$$
\sum_{k=0}^{\infty} N_{k}\left(l_{k}\right)^{n} \leq \sum_{k=0}^{\infty}\left(f^{k} \alpha^{n k}+1\right) \alpha^{-n k}=\sum_{k=0}^{\infty} f^{k}+\sum_{k=0}^{\infty}\left(\alpha^{-n}\right)^{k}=\frac{1}{1-f}+\frac{1}{1-\alpha^{-n}}<\infty .
$$

Hence, since $f<1$, we can fit everything into a bounded set as before. Clearly, $\Omega_{0}$ has again a smooth boundary. Its perimeter is also finite, since

$$
\begin{aligned}
P\left(\Omega_{0}\right) & =C \sum_{k=0}^{\infty} N_{k}\left(r_{k}\right)^{n-1} \leq C \sum_{k=0}^{\infty}\left(f^{k} \alpha^{n k}+1\right) r_{k} \\
& =\frac{C}{4}\left(\sum_{k=0}^{\infty} f^{k}+\sum_{k=0}^{\infty}\left(\alpha^{-n}\right)^{k}\right)=\frac{C}{4}\left(\frac{1}{1-f}+\frac{1}{1-\alpha^{-n}}\right) .
\end{aligned}
$$

On the other hand, we still find that balls at level $k$ have not yet hit anything else until time

$$
t_{k}=\frac{l_{k}}{2}-r_{k}=\frac{\alpha^{-k}}{2}-\frac{\alpha^{-k n}}{4} \geq \frac{\alpha^{-k}}{2}-\frac{\alpha^{-k}}{4}=\frac{\alpha^{-k}}{4} .
$$

Thus, for $t>0$ with $t_{m+1} \leq t<t_{m}$, we know that

$$
\begin{aligned}
P\left(\Omega_{t}\right) & \geq C \sum_{k=0}^{m} N_{k}\left(r_{k}+t\right)^{n-1} \geq C\left(t_{m+1}\right)^{n-1} \sum_{k=0}^{m} N_{k} \geq C\left(\frac{\alpha^{-(m+1)}}{4}\right)^{n-1} \cdot \sum_{k=0}^{m}\left(f \alpha^{n}\right)^{k} \\
& =\frac{C}{4^{n-1}}\left(\alpha^{n-1}\right)^{-(m+1)} \frac{f^{m+1}\left(\alpha^{n}\right)^{m+1}-1}{f \alpha^{n}-1} \geq \frac{C}{4^{n-1}\left(f \alpha^{n}-1\right)}\left((f \alpha)^{m+1}-1\right)=C^{\prime}\left(\left(\alpha^{s}\right)^{m+1}-1\right),
\end{aligned}
$$

where we have defined the constant $C^{\prime}$ suitably. From $(27)$, it follows that

$$
\alpha^{m+1} \geq \frac{1}{4 t_{m+1}} \geq \frac{1}{4 t} \Leftrightarrow\left(\alpha^{s}\right)^{m+1} \geq \frac{4^{-s}}{t^{s}} .
$$

Combining this with the estimate for $P\left(\Omega_{t}\right)$ above shows the claim.

Thus, we know from Corollary 1 that the rate of divergence of $P\left(\Omega_{t}\right)$ for $t \rightarrow 0^{+}$can be at most $1 / t$. The other way round, however, Theorem 5 shows that the rate can actually be worse than $1 / t^{s}$ for any $s \in(0,1)$.

\section{Acknowledgements}

The author would like to thank Wolfgang Ring of the University of Graz for thorough proof-reading of the manuscript. This work is supported by the Austrian Science Fund (FWF) and the International Research Training Group IGDK 1754.

\section{References}

[1] Luigi Ambrosio, Nicola Fusco, and Diego Pallara. Functions of Bounded Variation and Free Discontinuity Problems. Oxford Mathematical Monographs. Oxford Science Publications, Oxford, first edition, 2000.

[2] L. E. Blumenson. A Derivation of $n$-Dimensional Spherical Coordinates. The American Mathematical Monthly, 67(1):63-66, January 1960.

[3] Dorin Bucur and Jean-Paul Zolésio. Free Boundary Problems and Density Perimeter. Journal of Differential Equations, 126:224-243, 1996.

[4] Martin Burger. A framework for the construction of level set methods for shape optimization and reconstruction. Interfaces and Free Boundaries, 5:301-329, 2003. 
[5] Martin Burger, Norayr Matevosyan, and Marie-Therese Wolfram. A Level Set Based Shape Optimization Method for an Elliptic Obstacle Problem. Mathematical Models and Methods in Applied Sciences, 21(4):619-649, 2011.

[6] M. C. Delfour and J.-P. Zolésio. Shapes and Geometries. Advances in Design and Control. SIAM, second edition, 2001.

[7] Michel C. Delfour and Jean-Paul Zolésio. The New Family of Cracked Sets and the Image Segmentation Problem Revisited. Communications in Information and Systems, 4(1):29-52, September 2004.

[8] Marc Droske and Wolfgang Ring. A Mumford-Shah Level-Set Approach For Geometric Image Registration. SIAM Journal on Applied Mathematics, 66(6):2127-2148, 2006.

[9] Lawrence C. Evans and Ronald F. Gariepy. Measure Theory and Fine Properties of Functions. Studies in Advanced Mathematics. CRC Press, 1992.

[10] Herbert Federer. Geometric Measure Theory. Springer, 1969.

[11] Daniel Kraft. A Hopf-Lax Formula for the Time Evolution of the Level-Set Equation and a New Approach to Shape Sensitivity Analysis. Preprint IGDK-2015-18, https://igdk1754.ma.tum.de/ foswiki/pub/IGDK1754/Preprints/Kraft_2015A.pdf. Submitted to: Interfaces and Free Boundaries.

[12] Daniel Kraft. A Hopf-Lax Formula for the Level-Set Equation and Applications to PDE-Constrained Shape Optimisation. In Proceedings of the 19th International Conference on Methods and Models in Automation and Robotics, pages 498-503, 2014.

[13] S. Osher and J. A. Sethian. Fronts Propagating with Curvature-Dependent Speed: Algorithms Based on Hamilton-Jacobi Formulations. Journal of Computational Physics, 79:12-49, 1988.

[14] J. Yeh. Real Analysis: Theory of Measure and Integration. World Scientific, Singapore, second edition, 2006. 